\title{
TA-RELEVANTE BÜCHER
}

\section{EUROPEAN COMMISSION (Ed.): Green Paper on Environmental Issues of PVC. COM(2000)469, Brüssel, 2000}

\section{Rezension von Udo Jeske, Forschungszent- rum Karlsruhe, ITC-Zentralabteilung Tech- nikbedingte Stoffströme}

\section{$1 \quad$ Einleitung}

Im Juli 2000 wurde das Grünbuch-PVC zur Umweltproblematik von PVC von der EUKommission angenommen und veröffentlicht ${ }^{1}$. Damit wird die langjährig geführte Umweltdebatte um PVC auf die Gemeinschaftsebene gebracht. Die Motivation zur Erarbeitung einer Gemeinschaftsstrategie zu PVC speist sich aus verschiedenen, durchaus komplementären Quellen:

- aus der langjährigen kontroversen Diskussion in der Gemeinschaft um Umweltbelastungen durch die „Chlorchemie“, im speziellen

- aus Problemen durch die Verwendung von PVC und seinen Additiven in verschiedenen Phasen des Produktlebenszyklus komplex aufgebauter Produkte, sowie

- $\quad$ aus unterschiedlichen Maßnahmen einiger Mitgliedsländer $\mathrm{zu}$ speziellen Aspekten des Lebenszyklus von PVC, die Folgen für den Binnenmarkt haben könnten (Handelshemmnisse durch nationale Umweltschutzmaßnahmen).

In der Debatte um die Umweltwirkungen der Chlorchemie Ende der 80er und in den 90er Jahren wurde auch der Massenkunststoff PVC als Chlor-Großverbraucher einer kritischen Revision unterzogen. Von Umweltschützern wurde Druck auf die chlorverbrauchende Industrie insgesamt ausgeübt, von der Chlorbleiche bei der Papierherstellung, über Antiklopfmittel im bleihaltigen Benzin (Dioxinbildung) bis hin zu PVC, und es wurden die in unterschiedlichen Lebensphasen der Produkte bekannt gewordenen bzw. vermuteten Probleme kumuliert und „der“ Chlorchemie angelastet.
Daraus entwickelte sich das Ziel, diesen Zweig der Chemie zurückzudrängen zugunsten umweltfreundlicherer Alternativen. Die Chemische Industrie antwortete mit einer zunehmend flexiblen Strategie, in der zunächst grundsätzlich eine besondere Umweltbelastung durch PVC verneint, immer aber die Probleme vereinzelt und relativiert wurden, um dann in umstrittenen Bereichen dennoch zu reagieren und Korrekturen im Detail durchzuführen. Die Reaktionen erfolgten aufgrund gesetzlicher Vorgaben sowie auf der Basis „freiwilliger“ Vereinbarungen. Als Ziel der Industrie kann dabei ausgemacht werden, so viele Produkte der Chlorchemie wie möglich zu erhalten und deren Umsatz bei laufender Debatte auszuweiten. PVC ist hierfür geradezu ein Musterbeispiel.

\section{Das Grünbuch}

Das Grünbuch enthält von Anfang an Einschränkungen zum Untersuchungsbereich: Für Verpackungen (z. B. Blisterverpackungen im Pharmabereich), Kraftfahrzeuge (z. B. Armaturenbretter, Kabel, Unterbodenschutz) oder Elektro- und Elektronikschrott sind im Kontext der Debatte um nachhaltigere Produkte Fragen nach einer möglichen Substitutionspolitik für PVC-Produkte aus diesen Bereichen gestellt worden. Eine ersatzlose Streichung war bisher nicht durchsetzbar. Die weitere Untersuchung dieser Fragestellung würde deshalb auch die Analyse der Umweltwirkungen der möglichen Substitute über ihren Lebenszyklus erfordern. Eine solche Untersuchung war im Ansatz zum Grünbuch allerdings nicht vorgesehen.

Dennoch werden im Grünbuch folgende zu prüfende Fragen gestellt:

Welches sind die geeigneten Instrumente zur Entwicklung einer horizontalen Strategie zu PVC? Sollte für einige Produkte eine PVCSubstitutionspolitik ins Auge gefasst werden? Wenn ja, für welche?

Mit dem Grünbuch wurden von der Kommission zwei Ziele verfolgt:

- Umweltprobleme, die sich aus dem PVCEinsatz ergeben, sollten dargestellt und wissenschaftlich bewertet werden. Dabei erfolgte eine Konzentration auf PVCAdditive und die Abfallwirtschaft. 
- Mögliche Optionen einer Gemeinschaftsstrategie zu PVC sollten beschrieben werden, mit deren Hilfe diejenigen Umweltprobleme aus den untersuchten Bereichen reduziert werden können, für die Handlungsbedarf im Hinblick auf eine nachhaltige Entwicklung besteht.

Zur Vorbereitung waren Studien zu relevanten Umweltaspekten ,im Zusammenhang mit dem Vorhandensein von PVC in Abfallströmen“2 vergeben worden, in denen mechanisches Recycling, chemisches Recycling, Deponieren, Verbrennen sowie die Kosten der Abtrennung von PVC aus dem Abfallstrom untersucht wur$\mathrm{den}^{3}$.

\section{3}

\section{Untersuchte Umweltprobleme durch PVC}

PVC (Polyvinylchlorid) ist die Bezeichnung für einen Kunststoff auf Basis des gleichnamigen Polymers $\left(\mathrm{CH}_{2}=\mathrm{CHCl}\right)$. Das zugrunde liegende Monomer $\mathrm{VC}$ ist krebserzeugend (Kategorie 1), ebenso wie dessen Ausgangskomponente Ethylendichlorid (EDC) (Kategorie 2). Die Produktion findet in geschlossenen Anlagen statt und erfordert strenge Emissionskontrollen. Die benötigte Salzsäure $\mathrm{HCl}$ ist ätzend und reizend für die Atmungsorgane. Die Produktion von VC und PVC ist den Vorschriften mehrerer Gemeinschaftsrichtlinien unterworfen.

Nach der Polymerisation und nach ausreichender Entgasung des restlichen VC Monomers ist Roh-PVC außer als Staub nicht gesundheitsschädlich. Der Europäische Verband der PVC-Hersteller (ECVM) hat 1995 eine freiwillige Selbstverpflichtung abgegeben, nach der strenge Grenzwerte für eine Reihe von Stoffen festgelegt wurden, die mit der PVCProduktion einhergehen und die bis 1998 erfüllt werden sollten. Die Ziele wurden weitgehend, jedoch nicht vollständig erreicht. Eine erneute unabhängige Überprüfung ist für 2004 vorgesehen.

Die Weltproduktion von PVC liegt heute bei mehr als 20 Mio. $t$ pro Jahr und konzentriert sich auf USA, Westeuropa und Asien, mit Wachstumsraten zwischen 2 und $10 \%$ in den letzten Jahren. In Westeuropa lag die Produktion 1998 bei 5,5 Mio. t, für Europa werden derzeit 7,9 Mio. $t$ angegeben.
Das PVC-Polymer besteht zu $57 \%$ aus Chlor und ist deshalb ein vergleichsweise schwerer Kunststoff. Die hohen Bindungskräfte des Chlors im PVC-Polymer erleichtern die Zumischung einer großen Bandbreite eigenschaftsverändernder Additive. Dabei spielen Stabilisatoren und Weichmacher eine herausragende Rolle. Es sind im Abfallstrom neben dem Chlor gerade solche Additive, die Anlass zu bestehenden Umweltbesorgnissen geben.

\subsection{Stabilisatoren}

PVC braucht zum Schutz der Polymere vor dem Abbau durch Wärme und Licht Stabilisatoren. Risiken für Mensch und Umwelt gehen dabei von solchen Stabilisatoren aus, die Schwermetalle wie Blei und Cadmium enthalten. Über die tatsächliche Gefahr wird gestritten. In der Produktion gelten die Arbeitsschutzbestimmungen als ausreichend. In der Anwendungsphase sind die Stabilisatoren fest in den Kunststoff eingebunden. So verbleiben besonders die Abfallbeseitigung und der Brandfall.

Bei einem Gesamtverbrauch an Blei von 1,6 Mio. t (1995) in Europa fallen auf die Bleistabilisatoren etwa 51 Tausend t Blei (1998), die zu 112 Tausend t Bleistabilisatoren verarbeitet werden. Blei aus PVC-Stabilisatoren wird auf Anteile zwischen $1 \%$ und $28 \%$ am insgesamt im kommunalen Abfall befindlichen Blei geschätzt. PVC-Bleistabilisatoren sind nach Batterien und Akkumulatoren die bedeutendste industrielle Quelle für die Bleibelastung des Abfalls. Wird der Abfall verbrannt, so kann das Blei in hohen Anteilen an den Staub gebunden über die Rauchgasreinigung ausgeschleust werden, so dass eine akute Verbreitung des Stabilisatorbleis in der Umwelt auf diesem Weg nicht erfolgt. Auf der Hausmülldeponie als Alternative zur Verbrennung verhält sich PVC auch nach neueren Untersuchungen ausgesprochen stabil, so dass auch hier keine akute Kontamination, in diesem Falle des Sickerwassers, erfolgt. Übrig bleibt hier das langfristige Verhalten über die Lebenszeit der Deponieabdichtung hinaus.

Unter dem Druck der Umweltschützer, aber auch durch verbesserte Stabilisatorrezepturen ist der Einsatz von Cadmium für Stabilisatoren in Europa von 600 t pro Jahr (1992) auf 
50 t pro Jahr (1998) stark zurückgegangen. Die Industrie hat sich verpflichtet, die Verwendung von Cadmium-Stabilisatoren ab 2001 einzustellen. Das umfasst nicht die Importe aus Drittländern.

Als Alternative zu Blei wurden Stabilisatoren auf Basis Calcium/Zink und auf zinnorganischer Basis entwickelt. Insbesondere Calcium/Zink-Stabilisatoren sind derzeit nicht als gefährlich eingestuft. Nicht ganz so umweltgünstig wie Calcium/Zink verhalten sich die zinnorganischen Verbindungen.

Dänemark, Schweden, Österreich und Deutschland haben weitere - gesetzliche oder freiwillige - Einschränkungen der Verwendung von Blei als Stabilisatoren gefordert. Der generelle Ersatz von Bleistabilisatoren wird von der PVC-Industrie allerdings abgelehnt, wobei technische Faktoren (Produktqualität, Normen, Prüfanforderungen), wirtschaftliche Gründe (höhere Kosten) und das in den Augen der Industrie geringe Gefahrenpotenzial der Bleistabilisatoren ausschlaggebend sind. Die Vereinigung Europäischer Stabilisatorhersteller (ESPA) hat sich dennoch verpflichtet, erste Risikoabschätzungen zu Bleistabilisatoren bis 2004 durchzuführen, jährliche Statistiken über den Stabilisatoreinsatz vorzulegen sowie weiter an der Entwicklung alternativer Stabilisatoren $\mathrm{zu}$ arbeiten.

Vor diesem Hintergrund werden im Grünbuch folgende zu prüfende Fragen gestellt:

Welches Maßnahmenpaket sollte eingesetzt werden, um das Problem der Verwendung von Blei und Cadmium in neuem PVC anzugehen? Innerhalb welchen Zeitrahmens?

\subsection{Weichmacher}

PVC ist von Hause aus ein harter Kunststoff. Durch Zumischung von $10 \%$ bis $60 \%$, typisch $35 \%$ bis $40 \%$ Weichmacher zum Roh-PVC und einer anschließenden sogenannten „Compoundierung" kann aus dem harten Kunststoff ein weicher Kunststoff erzeugt werden. Etwa 1/3 der verkauften PVC-Produkte bestehen aus Weich-PVC. 1997 waren $93 \%$ der benötigten Weichmacher Phthalate (900 Tausend $\mathrm{t}$ von in Westeuropa insgesamt hergestellten 1 Mio. $t$ Phthalaten pro Jahr). Alle verschiedenen Phthalate, die in PVC-Produkten in großen
Mengen verwendet werden, sind heute in der Umwelt weit verbreitet. Die Abbaubarkeit in der Abwasserbehandlung ist eingeschränkt. Besorgnisse reichen von möglichen Auswirkungen auf Leber und Niere sowie Hoden bis zu Störungen des endokrinen Systems.

Risikobewertungen der fünf wichtigsten Phthalate gemäß der Verordnung 793/93 über chemische Altstoffe sind bereits oder werden bis 2001 beendet. Ohne die Ergebnisse der Risikobewertungen abzuwarten soll in Schweden das bisher wichtigste Phthalat, DEHP, zurückgedrängt werden. In Dänemark soll die Verwendung von Phthalaten in den nächsten zehn Jahren um $50 \%$ reduziert werden. In Deutschland wird vom Umweltbundesamt die Substitution von Weich-PVC in solchen Produkten empfohlen, für die sichere Alternativen vorhanden sind.

Die PVC-Industrie bemüht sich dagegen nach Kräften, die Unbedenklichkeit der permanenten Verluste von Weichmachern aus PVC im jeweiligen Produkteinzelfall nachzuweisen. Dabei wird insbesondere darauf abgestellt, dass die praktisch aufnehmbaren Mengen zu klein seien, um Schäden hervorzurufen.

Vor diesem Hintergrund werden im Grünbuch folgende zu prüfende Fragen gestellt:

Sollen spezielle Maßnahmen bezüglich der Verwendung von Phthalaten als Weichmacher in PVC getroffen werden? Wenn ja, wann und mit welchen Instrumenten?

\subsection{Chlor}

PVC-Abfälle tragen derzeit im Durchschnitt zu $50 \%$ des Chloreintrags in Verbrennungsanlagen bei. Weitere Chloreinträge stammen z. B. aus der Chlorbleiche von Papier, aber auch aus gesalzenen Küchenabfällen. Bei der Verbrennung von PVC in Müllverbrennungsanlagen wird Chlor in Form von Salzsäure frei. Einige Betreiber vertreten die Ansicht, dass der Dampfdruck und damit die Energieeffizienz für Abfallströme mit geringerem Chlorgehalt höher sein könnten. Erhöhte Korrosion der Anlagen erfordert einen schnelleren Wechsel von Komponenten und damit indirekt zusätzliche Umweltbelastungen aus der Komponentenherstellung. Die erforderliche Neutralisation des Chlorwasserstoffs führt zu Rückstandsmengen 
von (je nach Verfahren und PVC-Sorte) 0,5 bis $1,4 \mathrm{~kg}$ Rückständen pro kg PVC und damit zusätzlichen Kosten, die allerdings erst signifikant bei Vollauslastung der Verbrennungsanlage werden. Solange dies nicht der Fall ist, fährt PVC auf den allgemeinen Betriebskosten der Anlagen huckepack.

Beim gegenwärtigen Gehalt an Chlor von weniger als $1 \%$ am gesamten Massenstrom in die Verbrennungsanlage wird wissenschaftlich kein Zusammenhang (mehr) zwischen ChlorKonzentration und Dioxinbildung gesehen. Es wird diskutiert, ob eine Erhöhung des Chlorgehaltes im Abfallstrom über eine bestimmte Schwelle hinaus zu einer verstärkten Dioxinbildung führen kann. Dies könnte der Fall sein, wenn vermehrt langlebige PVC-Produkte, insbesondere aus dem Baubestand, in den Abfallstrom gelangen.

Bei unkontrollierten Bränden führt das Chlor sowohl zu Korrosionsschäden z. B. an Beton, als auch zu Dioxinbildung. Das Dioxin ist überwiegend an den Brandruß gebunden, der auch aus anderen Gründen gesundheitsgefährlich ist. Eine besonders hohe Dioxinbelastung durch unkontrollierte PVC-Verbrennung konnte bisher nicht festgestellt werden.

Vor diesem Hintergrund wird im Grünbuch folgende zu prüfende Frage gestellt:

Welcher Maßnahmenkatalog würde die mit der Verbrennung von PVC-Abfall zusammenhängenden Probleme am effektivsten ausräumen?

\section{$4 \quad$ Abfallwirtschaft}

Zwischen der PVC-Produktion und dem Erscheinen im Abfallstrom kann bei PVCProdukten, die im Bausektor eingesetzt werden, eine Zeitdifferenz von 10 bis 50 Jahren liegen. So ist, bedingt durch die bisherige Produktionsgeschichte, erst ab 2010 mit einem bedeutenden Ansteigen der Abfallmengen zu rechnen. Erwartet wird weiter, dass sich die $\mathrm{Zu}$ sammensetzung des Nach-Verbrauch-Abfalls von gegenwärtig zwei Drittel Weich-PVC und ein Drittel Hart-PVC in Richtung Hart-PVC verschiebt.

\subsection{Werkstoffliches Recycling}

Während für harte Profile aus PVC einerseits signifikante werkstoffliche Recyclingmöglichkeiten mit potenziell 50 - $70 \%$ Rezyklatanteil an der Neuware bestehen, verhält sich andererseits gerade Hart-PVC auf der Deponie besonders stabil, weshalb eine Ablagerung von der PVC-Industrie für unbedenklich gehalten wird. Sollten auch zukünftig die Möglichkeit zur Deponierung bestehen und die Deponie-Kosten niedrig bleiben, würde dies das werkstoffliche Recycling auch weiterhin schwächen. Gegenwärtig werden gerade einmal $3 \%$ der PVCGesamtmenge werkstofflich rezykliert. Die großen Mengen werden deponiert oder verbrannt. Werkstoffliches Recycling hat für eine Reihe von Hart-PVC-Produkten, wie Fensterprofile und Rohre ökologische Vorteile gegenüber der Frischproduktion. Aber die gegenwärtigen wirtschaftlichen Vorteile der Frischproduktion verhindern die mögliche Umweltentlastung durch verstärktes werkstoffliches Recycling. Zudem beklagt die PVC-Industrie, dass sie gegenwärtig keine größeren Abfallmengen sortenrein erfassen und dem Recycling zuführen könne.

In den für die Kommission angefertigten Studien zur Vorbereitung des Grünbuchs wird jedoch davon ausgegangen, dass bestehende oder künftige Richtlinien über Abfalldeponien, Verbrennung, Verpackungsmaterial, Altfahrzeuge, sowie Elektro- und Elektronikschrott umgesetzt werden. In der Folge wird mit steigenden Preisen für Verbrennung und Deponie gerechnet. Dadurch würden grundsätzlich die Bedingungen für die stoffliche PVCVerwertung verbessert.

Im Grünbuch wird festgestellt:

„Wenn eine wirtschaftliche Rentabilität nicht $\mathrm{zu}$ erreichen ist, findet unter den Bedingungen des freien Marktes kein PVC-Recycling statt, es sei denn, dies wird durch gesetzliche oder freiwillige Verpflichtungen erzwungen bzw. gefördert. Die Sammlung stellt den größten Engpass im Hinblick auf die Verfügbarkeit von Abfällen und die Kosten dar“. 
Vor diesem Hintergrund werden im Grünbuch folgende zu prüfende Fragen gestellt:

Mit welchem Maßnahmenkatalog ließe sich das Ziel einer stärkeren Nutzung des PVCRecyclings am effektivsten erreichen?

Sollte das werkstoffliche Recycling von bleiund cadmiumhaltigem PVC-Abfall an spezielle Bedingungen geknüpft werden? Wenn ja, an welche?

\subsection{Chemisches Recycling}

Die PVC-Industrie unternimmt eine Reihe von Versuchen, Verfahren für chemisches Recycling von PVC-haltigen Abfallfraktionen $\mathrm{zu}$ entwickeln. So wird bei der Verbrennung z. B. Chlorwasserstoff oder wieder Kochsalz erzeugt, die in den Produktionskreislauf zurückgeführt werden können. Alle bisherigen Verfahren, die auf eine Zerlegung der Polymermoleküle hinauslaufen, erwiesen sich als bei weitem zu teuer, um PVC gegenüber konkurrierenden Produkten auf Dauer wettbewerbsfähig zu halten. Der Nutzen besteht für die PVCIndustrie dennoch darin, gegenüber der Öffentlichkeit mit praktikablen Verfahren aufwarten zu können und die mangelnde Umsetzung auf fehlende Rahmenbedingungen schieben $\mathrm{zu}$ können.

In einer neuen Entwicklung werden sortenrein gesammelte PVC-Abfälle (Abdeckplanen, Kabel, pharmazeutische Blisterverpackungen, Bodenbeläge oder Armaturenbretter) durch selektive Lösung in PVC und die übrigen Komponenten getrennt. Danach wird PVC durch Ausfällung regeneriert und kann, wie auch andere enthaltenen Komponenten, wiederverwendet werden. Eine Pilotanlage soll 2001 fertiggestellt werden. Auch für dieses Verfahren gilt, dass die sortenreine Sammlung zunächst das Nadelöhr ist, das sowohl die verfügbaren Mengen begrenzt, als auch nicht unerheblich in die Recyclingkosten eingeht.

Vor diesem Hintergrund wird im Grünbuch folgende zu prüfende Frage gestellt:

Welcher Katalog von Maßnahmen wäre am geeignetsten für das chemische Recycling von PVC-Abfall?

\subsection{Deponierung}

Abfalldeponien müssen in der Gemeinschaft $a b$ 2001 überall technische Mindeststandards zum Schutz von Boden, Wasser und Atmosphäre erfüllen und deren Emissionen kontrollieren durch Basisabdichtung und Sickerwasser- sowie Gassammlung. Bei Fortschreibung der jetzigen Anteile an den Verwertungswegen wird von 2,8 Mio. $\mathrm{t} \mathrm{zu}$ deponierenden PVCAbfällen ausgegangen mit einem RecyclingAnteil von $9 \%$ der Nach-Verbrauchsabfälle, die insgesamt zu 6.2 Mio. t abgeschätzt wurden.

Unter Deponiebedingungen verhält sich Hart-PVC langzeitstabil, während bei WeichPVC permanent Phthalate entweichen und unter bestimmten Umständen dabei auch bis zu $10 \%$ der Stabilisatoren freigesetzt werden.

Vor diesem Hintergrund wird im Grünbuch folgende zu prüfende Frage gestellt:

Sind mit Blick auf die Deponierung von PVCAbfällen spezielle Maßnahmen erforderlich? Wenn ja, welche?

\section{$5 \quad$ Ausblick}

In einem Dialogprojekt von Ende 1996 bis Ende 1998 mit einer mehrteiligen Workshopreihe begleitete eine von der deutschen Arbeitsgemeinschaft PVC und Umwelt (AgPU) initiierte und finanzierte „Steuerungsgruppe“, mit Vertretern aus PVC-Industrie, UmweltWissenschaft, Journalisten, einem Umweltverband und Behörden die Erstellung einer Studie der Prognos AG, Basel zu ausgewählten PVCProduktsystemen ${ }^{4}$. Mit diesem Projekt wurde die Strategie der PVC-Industrie weiter verfeinert.

Im März 2000 unterzeichnete die gesamte europäische PVC-Industrie ${ }^{5}$ eine freiwillige Selbstverpflichtung, um ,unter Verwendung eines integrierten Ansatzes das Konzept eines verantwortlichen Managements von der Wiege bis zur Bare umzusetzen“.

Vor dem Hintergrund der Anforderungen an derartige Selbstverpflichtungen ${ }^{6}$ erfordert der Erfolg dieses Ansatzes das Vorhandensein bzw. die realistische Entwicklung tatsächlicher Möglichkeiten, signifikante Umweltentlastungsziele zu erreichen. Zur Beurteilung dienen dazu: 
- die Reduzierung der Produktion und der Verwendung bestimmter Additive

- die Festsetzung anspruchsvoller Mengenziele für das Recycling

- der Beitrag der Industrie zu den Mehrkosten der Müllverbrennung

- ein voll funktionsfähiger Finanzierungsmechanismus.

Die Öffentlichkeit war zur Diskussion und Kommentierung des Grünbuchs bis Ende November 2000 eingeladen. Die eingegangenen 35 Stellungnahmen wurden über das Internet zugänglich gemacht. Ein öffentliches Hearing fand im Oktober 2000 statt. Mit der so geschaffenen Basis will die Kommission Anfang 2001 eine umfassende Gemeinschaftsstrategie zu den Umweltaspekten von PVC festlegen.

Über die Ergebnisse der öffentlichen Anhörung wird noch berichtet.

\section{Anmerkungen}

1) http://europa.eu.int./comm/environment/pvc/in dex.htm

2) Europäische Kommission (Hrsg.): Vorschlag für eine Richtlinie des Rates über Altfahrzeuge. $\operatorname{KOM}(97) 358$ endg.

3) http://europa.eu.int./comm/environment/waste/ facts_en.htm

4) Steuerungsgruppe zum Dialogprojekt PVC und Nachhaltigkeit und Arbeitsgemeinschaft PVC und Umwelt (Hrsg.), 1999: PVC und Nachhaltigkeit. Köln: Dt. Instituts-Verlag. ISBN 3-602-14485

5) Hersteller: ECVM; Weichmacher und Zwischenprodukte: ECPI; Stabilisatoren: ESPA; Verarbeiter: EuPC

6) Europäische Kommission (Hrsg.): Mitteilung der Kommission an den Rat und das Europäische Parlament über Umweltvereinbarungen. $\operatorname{KOM}(96) 561$ endg.

\section{„Human Choice \& Climate Change“ - Ein sozialwissenschaftliches As- sessment}

\author{
Rezension des vierbändigen Kompendiums \\ von S. Rayner, E.L. Malone: „Human Choice \\ and Climate Change" von Silke Beck, ITAS
}

\section{Die Forschungslandschaft}

Um das Kompendium einzubetten, ist einleitend der Blick auf die Forschungslandschaft zu werfen. Ab Mitte der 80er Jahre gewinnen die humanen Dimensionen (human dimensions) der Klimaproblematik in der Forschung an Gewicht. Im Hinblick auf die Integration humaner Dimensionen sind in aller gebotenen Kürze folgende Sachverhalte festzuhalten: Auf der einen Seite wird der „Menschheit“ als Verursacherin des Klimawandels eine gewichtige Rolle beigemessen. Auf der anderen Seite wird das globale Klima bis dato weitgehend als natürliches System behandelt. Die humanen Dimensionen werden mehr oder weniger auf einen Faktor reduziert, der ausschließlich als eine weitere, kritische Variable in die naturwissenschaftlichen Modelle integriert wird. Der Beitrag der Sozialwissenschaften beläuft sich darauf, den Input in die naturwissenschaftlichen Modelle zu liefern. In diesem Szenarium kommt sozialwissenschaftlicher Forschung nicht nur die Rolle des Nachzüglers, sondern des Lückenfüllers zu. Ihr Beitrag beläuft sich darauf, die von den Naturwissenschaften auferlegten Hausaufgaben zu erledigen.

Die Architektur der neueren Klimaforschung legt den Vergleich mit einer WissensPyramide nahe. Zentrales Kriterium ist die Härte der Wissenschaften. Naturwissenschaften gelten als harte, Sozialwissenschaften hingegen als weiche Wissenschaften. Diese Hierarchisierung von Wissenschaften nach ihrer vermeintlichen Härte erklärt nicht nur, warum Sozialwissenschaften eine nachgeordnete Rolle spielen, sondern auch, warum die am naturwissenschaftlichen Ideal (der science) orientierten Disziplinen wie Ökonomie und Psychologie die sozialwissenschaftliche Diskussion dominieren. 